Amatria, M.; Lapresa, D.; Martín Santos, C.; Pérez Túrpin, J.A. (2020) Offensive Effectiveness in the Female Elite Handball in Numerical Superiority Situations. Revista Internacional de Medicina y Ciencias de la Actividad Física y el Deporte vol. 20 (78) pp. 227-242 Http://cdeporte.rediris.es/revista/revista78/artanalisis1161.htm

DOI: http://doi.org/10.15366/rimcafd2020.78.003

\title{
ORIGINAL
}

\section{EFICACIA OFENSIVA EN EL BALONMANO FEMENINO DE ÉLITE EN SITUACIONES DE SUPERIORIDAD NUMÉRICA}

\section{OFFENSIVE EFFECTIVENESS IN FEMALE ELITE HANDBALL IN NUMERICAL SUPERIORITY SITUATIONS}

\author{
Amatria, M. ${ }^{1}$; Lapresa, D. ${ }^{2}$; Martín Santos, C. ${ }^{3}$ y Pérez Túrpin, J.A. ${ }^{4}$ \\ 1 Profesor Adjunto, Facultad de Educación. Universidad Pontificia de Salamanca (España). \\ mamatriaii@upsa.es \\ 2 Profesor Titular, Facultad de Letras y de la Educación. Universidad de la Rioja (España) \\ daniel.lapresa@unirioja.es \\ 3 Ldo. Cc. de la Actividad Física y Deporte. Universidad Pontificia de Salamanca (España) \\ carlos santos91@hotmail.com \\ 4 Profesor Titular, Facultad de Educación. Universidad de Alicante (España) \\ jose.perez@gcloud.ua.es
}

Código UNESCO / UNESCO code: 5899 Otras especialidades pedagógicas (Educación Física y Deporte).

Clasificación Consejo de Europa/Council of Europe classification: 4. Educación Física y deporte comparado.

Recibido 27 de julio de 2018 Received July 27, 2018

Aceptado 16 de agosto de 2019 Accepted August 16, 2019

\section{RESUMEN}

El balonmano, a pesar de ser uno de los deportes más practicados en Europa, no despierta gran interés por parte de la comunidad científica en su modalidad femenina. Dada la relevancia que tienen en los partidos las situaciones de superioridad numérica, el presente trabajo tiene como objetivo analizar las secuencias ofensivas, eficaces e ineficaces, que se producen en el balonmano femenino de élite en situaciones de superioridad numérica ofensiva, durante el Campeonato Mundial celebrado en Suecia en 2016. Para ello se ha hecho uso de la metodología observacional y del análisis secuencial de retardos. Los resultados obtenidos revelan como patrón de juego ofensivo exitoso, es decir, que la secuencia ofensiva concluye en gol, un patrón de juego que utiliza un sistema táctico no estructurado, con independencia de su forma de inicio, que 
emplea como principal medio ofensivo el contraataque de primera oleada, para finalizar las acciones con situaciones de $1 \mathrm{vs} 0$.

PALABRAS CLAVE: balonmano femenino, balonmano, metodología observacional, ataque.

\begin{abstract}
Despite being one of the most practiced sports in Europe, handball does not generate great interest on the part of the scientific community in its female modality. Given the relevance of situations of numerical superiority during the matches, the present work aims to analyze, in greater detail, the effective and ineffective offensive superiority sequences that occurred during the Women Handball World Championship (Sweden 2016). The observational methodology and the sequential analysis of delays have been used for this investigation. The results obtained reveal a successful offensive game pattern (ending in goal). This pattern uses an unstructured tactical system, regardless of its starting form, using the first wave counterattack as the main offensive play, to finish the actions with situations of $1 \mathrm{vs} 0$.
\end{abstract}

KEY WORDS: female handball, handball, observational methodology, attack.

\title{
INTRODUCCIÓN
}

La práctica del balonmano ha evolucionado en los últimos años: los jugadores y cuerpo técnico han alcanzado un mayor nivel de profesionalización; los medios de entrenamiento han evolucionado en la búsqueda del rendimiento; y las competiciones se han convertido en campeonatos con un elevado nivel de juego (Prudente, Sousa, Sequeira, López-López y Hernández-Mendo, 2017).

En la actualidad, el rendimiento de los diferentes conjuntos que compiten en los torneos internacionales más relevantes (Campeonatos del Mundo y de Europa) ha alcanzado cotas muy semejantes entre sí; dependiendo la victoria en estos partidos de pequeños detalles que jalonan el desarrollo de los encuentros (Ferrari, dos Santos y Vaz, 2014). En este sentido, el reglamento ha sufrido diversas modificaciones con la intención de hacer este deporte más espectacular y favorecer la existencia de esos pequeños detalles que desequilibren la balanza y hagan del balonmano un espectáculo más imprevisible y emocionante.

El balonmano es uno de los deportes más practicados de Europa (Prieto, Gómez y Sampaio, 2015) y, consecuentemente, es fuente de interés de la comunidad científica desde diversas facetas: biomédica (Asker, Waldén, Källberg, Holm y Skillgate, 2017; Fieseler, Hermassi, Hoffmeyer, Schulze, Irlenbusch, Bartels, Delank, Laudner y Schwesig, 2017; Grabara, 2018; Lubiatowski, Kaczmarek, Cisowski, Breborowicz, Grygorowicz, Dzianach, Krupecki, Laver y Romanowski, 2018; Pueo, Jiménez-Olmedo, Penichet-Tomas, Ortega y Espina, 2017), psicológica (Mayer y Thiel, 2018), del análisis del juego o match analysis (Cardinale, Whiteley, Hosny, Popovic, 2017; Gutiérrez, Fisette, García-López y 
Contreras, 2014; Navarro, Morillo, Reigal y Hernández-Mendo, 2018), relacionadas con la preparación física y el entrenamiento (Debanne, Laffaye y Trouilloud, 2018; Dello lacono, Martone, zagatto, Meckel, Sindiani, Milic y Padulo, 2018; Forthomme, Croisier, Delvaux, Kaux, Crielaard y Gleizes-Cervera, 2018; Hermassi, Chelly, Fieseler, Bartels, Schulze, Delank, Shepard y Schwesig, 2017; Menezes, dos Reis y Tourinho, 2015; Sabido, Hernández-Davo, Botella, Navarro y Tous-Fajardo, 2017; Srhoj, Rogulj, Padovan, y Katić, 2001; Wagner, Gierlinger, Adzamija, Ajayi, Bacharach, y von Duvillard, 2017).

El balonmano femenino no despierta tanto interés como el masculino, lo que constituye un reflejo del camino que todavía le resta por recorrer al deporte femenino en pos de la igualdad de consideración (Lopiano, 2000). Es por ello que la producción en las referidas facetas es menor: aspectos biomédicos (Ntai, Zahou, Paradisis, Smirnitou \& Tsolakis, 2017; Steffen, Nilstad, Krosshaug, Pasanen, Killingmo \& Bahr, 2017), relacionados con la preparación física y el entrenamiento (Mhenni, Michalsik, Mejri, Yousfi, Chaouachi, Souissi \& Chamari, 2017; Ohnjec, Vuleta, Milanović y Gruić, 2008), iniciación deportiva (Ingebrigtsen, Jeffreys \& Rodahl,, 2013), y el análisis del juego o match analysis (Michalsik, Aagaard y Madsen, 2013; Morillo-Baro, Reigal y Hernández-Mendo, 2015).

Dentro del match analysis, se engloba el estudio de la asimetría numérica durante el desarrollo del juego. Una situación derivada del incumplimiento de las reglas de juego, en el que uno de los equipos tiene una ventaja numérica -tanto defensiva como ofensivamente- respecto a su adversario (Espina, Pérez y Cejuela, 2011; Gutiérrez, Fernández y Borrás, 2010). Estas situaciones de asimetría numérica favorable, favorecen la consecución del éxito en la secuencia de juego (Wagner, Finkenzeller, Würth y von Duvillard, 2014). Siendo conscientes de la relevancia del gol en este deporte, el estudio y conocimiento de las situaciones previas a su consecución adquiere una importancia manifiesta para entrenadores y jugadores por su alta repercusión en la obtención de rendimiento en el juego (Hristovki, Aceski, Balague, Seifert, Tufeckcievski y Cecilia, 2017).

De esta forma, y conforme lo anteriormente expuesto, el presente trabajo persigue el objetivo de analizar, en el balonmano femenino de élite, la construcción de las secuencias ofensivas, eficaces e ineficaces, en situaciones de superioridad numérica favorable.

\section{MÉTODO}

Este estudio se desarrolla en el seno de la metodología observacional (Anguera, 1979), que ha encontrado en el deporte -por la cantidad y variedad de conductas susceptibles de ser observadas-, un entorno propicio para su desarrollo metodológico (Anguera y Hernández-Mendo, 2015). También los estudiosos del balonmano han acudido a la metodología observacional por las posibilidades que brinda al análisis del juego (González, 2015; Morillo y Hernández-Mendo, 2015; Prudente et al., 2017; Ribeiro, Oliveira, Freitas, Leitao, Anguera y Campaniço, 2009). 
El diseño observacional seguido es, de acuerdo con Anguera, Blanco-Villaseñor y Losada 2011: a) De seguimiento intersesional -23 partidos- e intrasesional frame a frame- en cada secuencia analizada; b) nomotético -los 12 equipos participantes- y c) multidimensional -como reflejan las cinco dimensiones que vertebrarán, posteriormente, el instrumento de observación-.

\section{Participantes}

Forman parte del presente estudio todos los equipos clasificados para el desarrollo de la segunda fase -main round- y siguientes eliminatorias de clasificación del Campeonato Mundial de balonmano femenino que tuvo lugar en Suecia, 2016 siendo un total de 12 equipos. El muestreo observacional secuencias ofensivas en superioridad por exclusión de jugadoras del equipo rival- se ha extraído de la totalidad de los partidos disputados en el main round y las posteriores eliminatorias de las que consta el campeonato -23 partidos-. La celebración de los campeonatos del mundo femeninos de balonmano se celebran durante los meses finales del año, habitualmente diciembre, por lo que la recuperación de todos los videos necesarios para poder desarrollar la investigación se produce ya en 2017. Esta recuperación de los videos viene marcada debido a la falta de emisiones de esta modalidad deportiva de forma pública "en abierto". Una vez se dispone de los videos, el registro de los datos, los análisis y la elaboración del artículo se realizan posteriormente, estando el documento preparado y enviado a la revista el 27 de julio de 2018 (únicamente se había celebrado el mundial de 2017 posterior al analizado), por lo que con los procesos de evaluación nos ha llevado hasta el día de la fecha.

A pesar de ello en la actualidad no existen estudios similares ni con las aportaciones realizadas por éste, siendo un fuerte punto de partida para investigaciones futuras tanto de carácter longitudinal (ya se están llevando a cabo por parte de este grupo de investigación) con los campeonatos siguientes como transversal.

Así mismo, el conocimiento de la generación de juego es imprescindible para entender el juego en sí mismo y mejorar en su rendimiento y práctica.

Por último, la investigación y avance científico en la modalidad femenina adquiere una relevancia capital hoy en día, ya que cada vez cobra un mayor auge en nuestra sociedad.

\section{Instrumento de observación}

Se ha elaborado un instrumento de observación ad hoc (tabla 1), para el registro de los datos, haciendo uso de la propuesta de Lozano (2014) para la dimensión "construcción". El instrumento de observación es una combinación de formato de campo y sistemas de categorías (Anguera, Magnusson y Jonsson, 2007); en el que todas las dimensiones del formato de campo se despliegan en sistemas de categorías al cumplir los requisitos de exhaustividad y mutua exclusividad (Anguera y Hernández-Mendo, 2013). 
Tabla 1. Instrumento de observación.

\begin{tabular}{|c|c|c|}
\hline № & Dimensión & Sistemas de Categorías: códigos y breve descripción \\
\hline 1 & $\begin{array}{l}\text { Posesión del } \\
\text { balón }\end{array}$ & $\begin{array}{l}\text { PO) Posesión por equipo observado; PC) Posesión por equipo contrario; Inob) } \\
\text { Inobservabilidad. }\end{array}$ \\
\hline 2 & $\begin{array}{l}\text { Inicio de la } \\
\text { fase ofensiva }\end{array}$ & $\begin{array}{l}\text { RO) Robo de balón en situaciones de } 1 \times 1 \text { o 1x2; IN) Interceptación del balón; SP) } \\
\text { Saque de puerta; SC) Saque de centro; ER) Infracción reglamentaria por parte del } \\
\text { equipo rival; PB) Pérdida de balón del equipo rival. }\end{array}$ \\
\hline 3 & $\begin{array}{l}\text { Tipología de } \\
\text { ataque }\end{array}$ & $\begin{array}{l}\text { CA) Contraataque; 65) ataque posicional en superioridad } 6 \times 5 ; 76 \text { ) ataque } \\
\text { posicional en superioridad } 7 \times 6 \text {; GF) Golpe franco; SE) situaciones especiales } \\
\text { superioridad numérica de dos o más jugadores. }\end{array}$ \\
\hline 4 & Construcción & STE) sistema táctico estructurado; STNE) sistema táctico no estructurado. \\
\hline 5 & $\begin{array}{l}\text { Zona de inicio } \\
\text { de jugada }\end{array}$ & $\begin{array}{l}\text { ZIA, ZIB, ZIC -sector primera línea-, ZID, ZIE, ZIF -sector segunda línea-; ZICP - } \\
\text { Campo propio- (véase figura 1). }\end{array}$ \\
\hline 6 & Creación & $\begin{array}{l}\text { CR) cruce; (CRP) cruce con pivote; PER) permuta; PEX) permuta exterior; Trns24) } \\
\text { transformación a sistema ofensivo } 2-4 \text {; BLO) bloqueo; PAN) pantalla; COR) } \\
\text { cortina; PV) pase y va; PS) penetraciones sucesivas; JA) juego aéreo; } \mathrm{X} 11 \text { ) acción } \\
\text { de } 1 \times 1 \text {; X22) acción de } 2 \times 2 \text {; CA1O) contrataque en primera oleada; CA2O) } \\
\text { contraataque en segunda oleada. }\end{array}$ \\
\hline 7 & $\begin{array}{l}\text { Segunda } \\
\text { Creación }\end{array}$ & $\begin{array}{l}\text { CR2) cruce; CRP2) Cruce con pivote; PER2) permuta; PEX2) permuta exterior; } \\
\text { Trns242) transformación a sistema ofensivo 2-4; BLO2) bloqueo; PAN2) pantalla; } \\
\text { DES2) Deslizamiento; PV2) pase y va; PS2) penetraciones sucesivas; JA2) juego } \\
\text { aéreo; X112) acción de } 1 \times 1 \text {; X222) acción de } 2 \times 2 \text {; NING) No se produce segunda } \\
\text { creación. }\end{array}$ \\
\hline 8 & Prefinalización & $\begin{array}{l}\text { VS43) situación de } 4 \text { contra 3; VS32) situación de } 3 \text { contra 2; VS21) situación de } \\
2 \text { contra 1; VS10) situación de } 1 \text { contra el portero, sin defensores. }\end{array}$ \\
\hline 9 & Finalización & $\begin{array}{l}\text { GOL) lanzamiento y gol; ML) lanzamiento que no acaba en gol; } 7 \mathrm{M} \text { ) sanción de } 7 \\
\text { metros a favor del equipo observado; FGF) golpe franco a favor del equipo } \\
\text { observado; PBL) Pérdida de balón por parte del equipo observado; ERR) comisión } \\
\text { de un error reglamentario por parte del equipo observado. }\end{array}$ \\
\hline 10 & $\begin{array}{l}\text { Zona de } \\
\text { finalización }\end{array}$ & $\begin{array}{l}\text { ZFA, ZFB, ZFC -sector primera línea-, ZFD, ZFE, ZFF -sector segunda línea-. } \\
\text { Véase figura } 1 .\end{array}$ \\
\hline
\end{tabular}

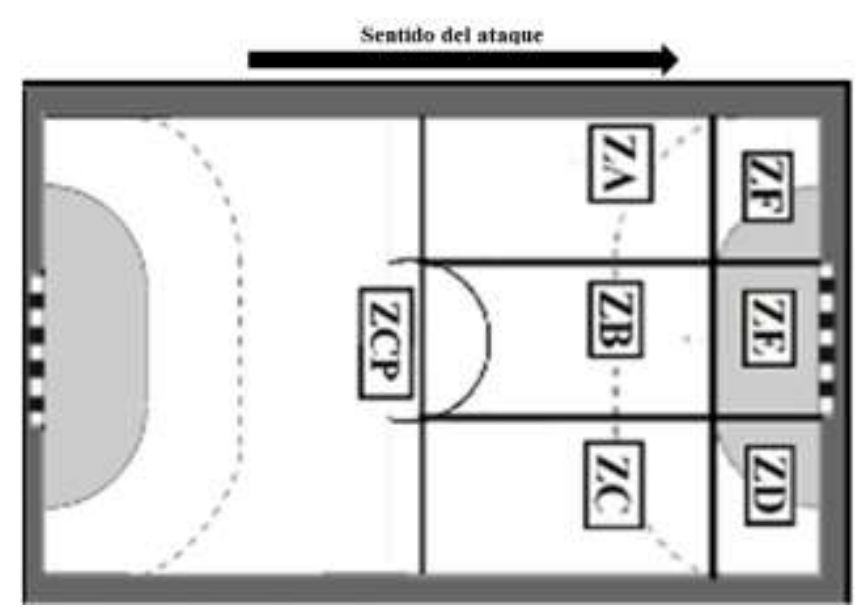

Distribución Zonal del espacio 


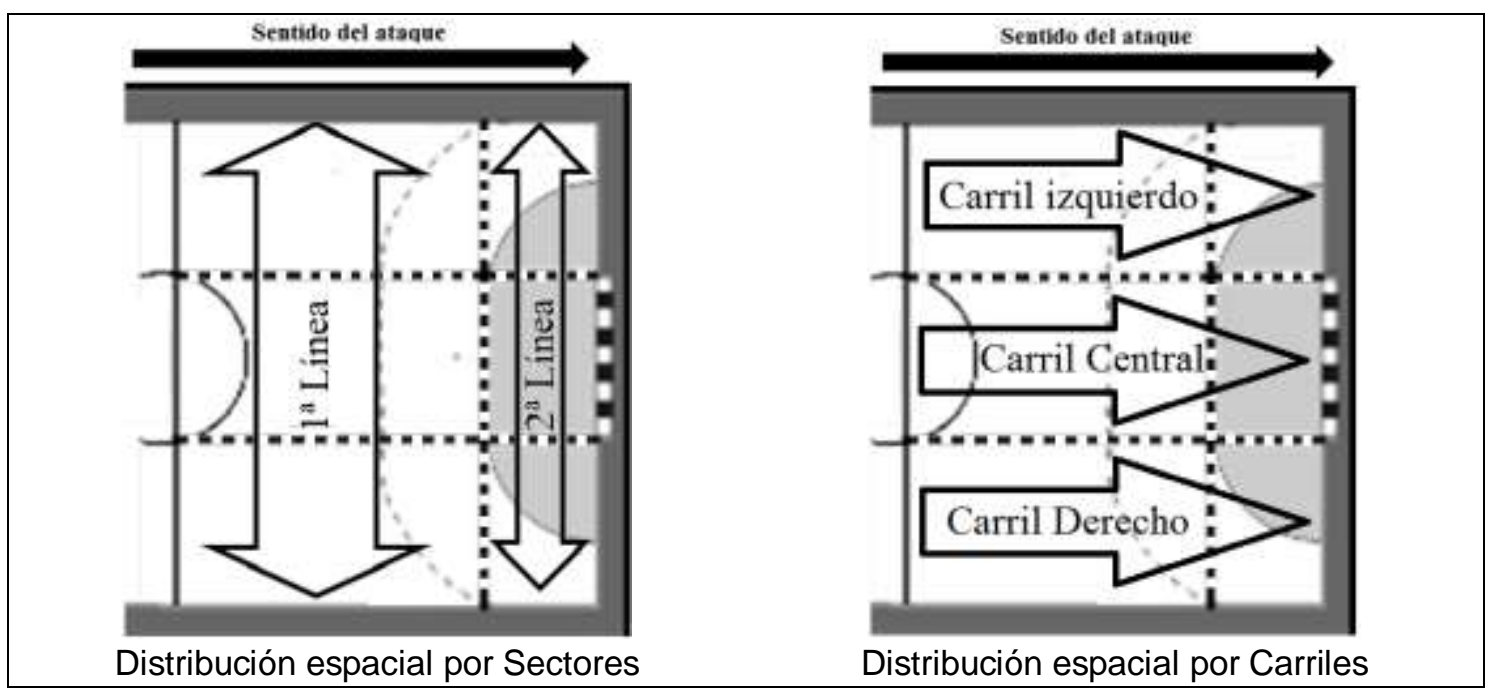

Figura 1. Campograma de distribución espacial atendiendo a zonas, sectores y carriles del terreno de juego.

\section{Registro y codificación}

El registro de los datos se ha realizado mediante el software Lince (Gabin, Camerino, Anguera y Castañer, 2012), versión 1.2.1. Se han registrado cada una de las secuencias ofensivas que se han producido en situaciones de superioridad numérica, por exclusión de una jugadora por parte del equipo rival, en segunda fase y siguientes eliminatorias de clasificación del Campeonato Mundial de balonmano femenino 2016. El registro de cada secuencia se realizó en tres fases: el inicio de la secuencia ofensiva; el desarrollo de la secuencia, compuesto como mínimo por una acción de creación; y, por último, el final de la secuencia véase figura 2-. Se han registrado un total de 262 secuencias ofensivas en superioridad por exclusión de jugadoras del equipo rival. De acuerdo con Bakeman y Quera (1995), los datos obtenidos son de multievento.

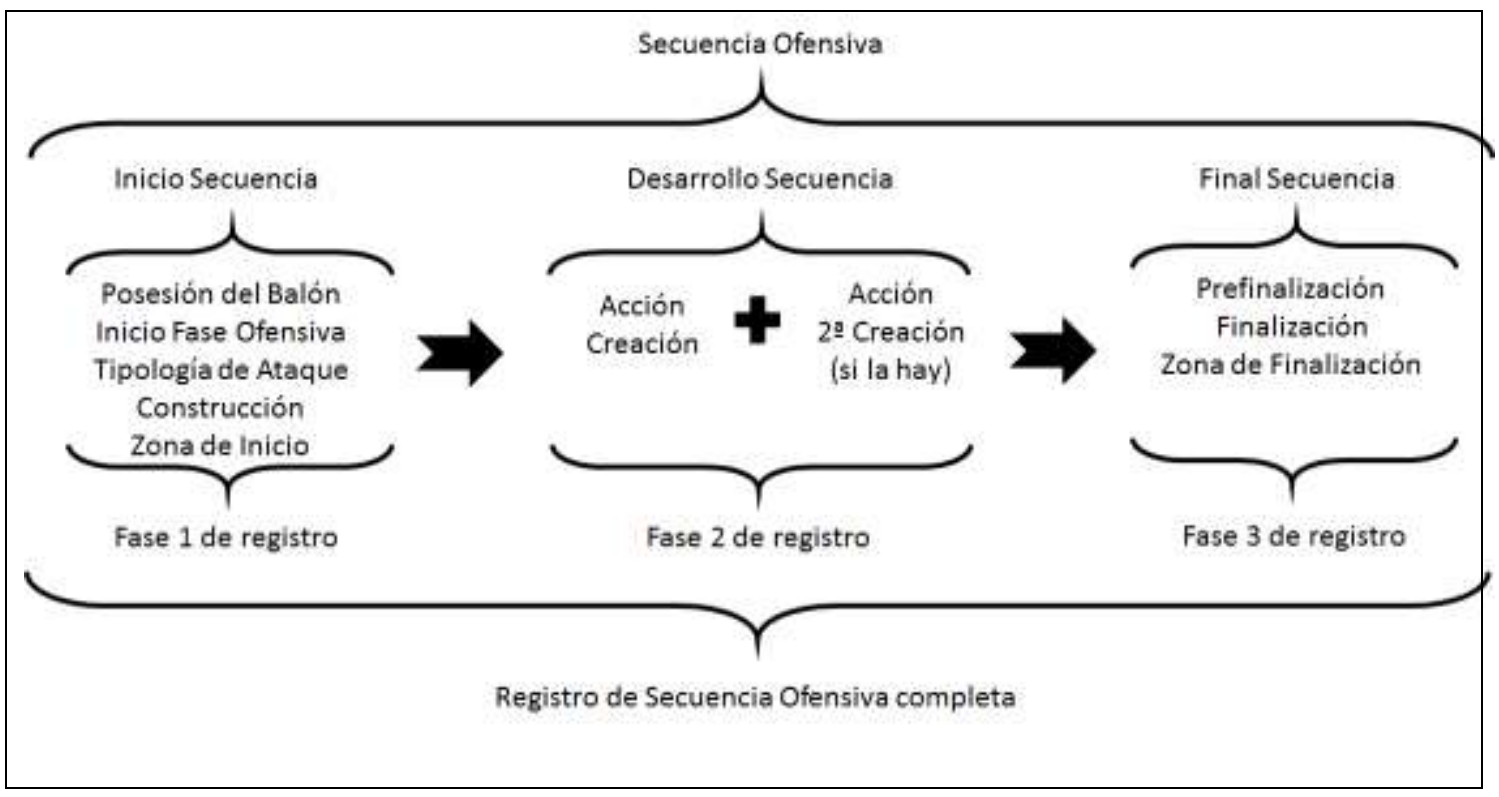

Figura 2. Estructura del registro de cada secuencia ofensiva: fases y dimensiones a registrar. 


\section{Fiabilidad del dato}

Los datos fueron registrados por dos observadores, licenciados en Ciencias de la Actividad Física y el Deporte, con experiencia en balonmano y metodología observacional, que previamente realizaron un proceso de formación a partir de Anguera (2003). Uno de los observadores registró todas las secuencias que constituyeron el muestreo observacional; mientras que el segundo observador registró el $10 \%$ del total.

Con el objeto de determinar la fiabilidad -en forma de concordancia- de los datos obtenidos a partir del instrumento de observación, se ha empleado el Kappa de Cohen (1960), mediante el programa GSEQ (Bakeman y Quera, 2011), versión 5.1. Se han calculado, por dimensiones o criterios del instrumento de observación, los valores del Kappa de Cohen correspondientes a los paquetes de datos registrados por ambos observadores; obteniéndose un valor mínimo de $\mathrm{k}=0,91$ (tabla 2), lo que garantiza la fiabilidad de los datos que soportan el presente trabajo (Fleiss, Levin y Paik, 2003).

Tabla 2. Concordancia entre observaciones: Kappa de Cohen por dimensiones del instrumento de observación.

\begin{tabular}{lc}
\hline Dimensión & Kappa de Cohen \\
\hline Posesión del balón & 1 \\
\hline Inicio de la fase ofensiva & 1 \\
\hline Tipología de ataque & 1 \\
\hline Construcción & 0,91 \\
\hline Zona de inicio de jugada & 0,93 \\
\hline Creación & 0,94 \\
\hline Segunda Creación & 0,91 \\
\hline Prefinalización & 0,95 \\
\hline Finalización & 0,93 \\
\hline Zona de finalización & 0,95 \\
\hline
\end{tabular}

\section{Análisis de los datos}

Se ha realizado un análisis diacrónico de los datos, para dar respuesta al objetivo del presente estudio. En concreto, se ha llevado a cabo un análisis secuencial de retardos mediante el software GSEQ, v 5.1 (Bakeman y Quera, 2011).

Se ha realizado el cálculo de los residuos ajustados entre las conductas criterio -given en terminología GSEQ- correspondientes a la dimensión finalización GOL, ML, 7M, FGF, PBL y ERR- y las conductas condicionadas -targetcorrespondientes a las dimensiones del instrumento: inicio de la fase ofensiva, tipología de ataque, construcción, zona de inicio de jugada, zona de finalización, creación, segunda creación, y pre-finalización. Se han analizado las conductas condicionadas retrospectivas relacionadas con las categorías criterio establecidas, desde el retardo -3 al retardo 0 o coocurrencia.

De acuerdo con Bakeman, y Gottman (1986), las transiciones mayores de 1,96 muestran una relación estadísticamente significativa $(p<0,05)$ de activación entre 
la conducta given y la conducta target; mientras que las transiciones menores de -1,96, señalan una relación estadísticamente significativa de inhibición entre la conducta criterio y la conducta condicionada).

\section{RESULTADOS}

Aprovechando las posibilidades que brinda la metodología observacional para capturar la conducta desplegada conforme los parámetros orden y duración, se ha realizado un análisis secuencial de retardos para dar respuesta al objetivo establecido, obteniéndose los resultados que se presentan en la tabla 2.

Como conductas criterio o given, se han establecido las correspondientes a la dimensión finalización de la acción ofensiva -GOL, ML, 7M, FGF, PBL y ERR- y como conductas condicionadas -o target- se han fijado las categorías correspondientes a las dimensiones: inicio de la fase ofensiva, tipología del ataque, zonas de inicio y final de la acción ofensiva, tipo de creación y segunda creación; y situación de pre-finalización. En función del objetivo de analizar, en el balonmano femenino de élite, la construcción de las secuencias ofensivas, eficaces e ineficaces, en situaciones de superioridad numérica favorable, el análisis secuencial de residuos ajustados se ha realizado en la vertiente retrospectiva (retardos $-3,-2,-1$ ) y, al ser datos de multievento, en la coocurrencia. 
Tabla 2. Residuos ajustados significativos que constituyen patrones secuenciales (de activación -superiores a 1,96- o inhibición -inferiores a -1,96-) de finalización de las secuencias ofensivas, en las situaciones de superioridad numérica favorable.

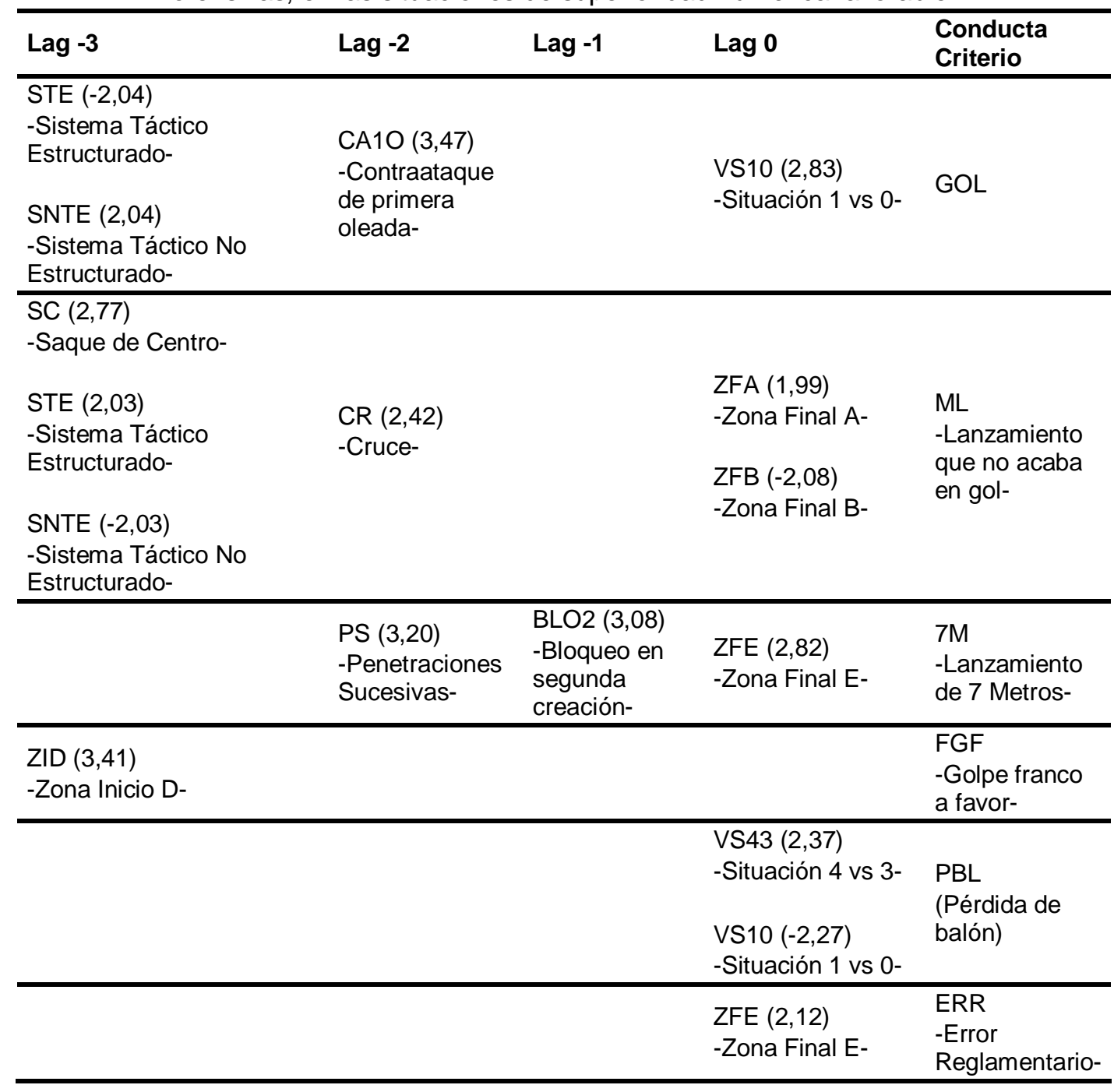

\section{DISCUSIÓN}

En el análisis secuencial de retardos, se han tomado como conductas criterio las relativas a la dimensión "finalización" (GOL, ML, 7M, GF, PBL, ERR), y como conductas condicionadas las correspondientes al resto de dimensiones que componen el instrumento de observación (inicio de la fase ofensiva, tipología de ataque, construcción, zona de inicio de jugada, zona de finalización, creación, segunda creación, y pre-finalización). Se consideran secuencias ofensivas exitosas aquellas cuya finalización de la acción ofensiva concluye en gol (GOL) o con la señalización de un lanzamiento de 7 metros (7M); las secuencias ofensivas se considerarán "no exitosas" si finalizan con un mal lanzamiento (ML), un golpe franco a favor (FGF), una pérdida de balón (PBL) o con la comisión de un error reglamentario durante la acción ofensiva por parte del equipo observado (ERR).

Los resultados obtenidos correspondientes a aquellas secuencias ofensivas de superioridad numérica ofensiva favorable que finalizan en gol (GOL), reflejan un 
patrón de conducta constituido por un sistema táctico no estructurado (STNE), empleando como elemento de creación en su desarrollo el contraataque de primera oleada (CA1O), concluyendo con situaciones de pre-finalización de 1vs0 (VS10) -es decir, del lanzador atacante contra el portero adversario-. Este patrón de conducta atiende a la lógica interna de un contraataque y está en sintonía con los resultados obtenidos por Hernández et al. (2010) que concluyen la mayor eficacia del contraataque, frente al juego posicional, de cara a la consecución de gol. Así mismo, coinciden con los obtenidos por Ribeiro et al. (2009), en su estudio comparativo entre contraataques y ataques rápidos en balonmano masculino -detectaron patrones temporales en las secuencias de contraataque directo, pero no en las de ataque rápido-. Por su parte, Lozano y Camerino (2012), en su análisis de la eficacia de los sistemas ofensivos en balonmano, concluyen que el contraataque es el tipo de acción ofensiva más eficaz independientemente del tipo de defensa desarrollada por el equipo adversario; a pesar de ello, difieren con los resultados obtenidos en esta investigación, al concluir que el sistema táctico más eficaz es el estructurado (STE).

En referencia a los resultados obtenidos correspondientes a aquellas secuencias ofensivas que, produciéndose en una situación de superioridad numérica ofensiva favorable, finalizan en un lanzamiento de 7 metros a favor (7M), manifiestan la presencia, de la categoría PS (penetraciones sucesivas) en primera creación y BLO2 (bloqueo en segunda creación) como segunda acción de creación, con valores positivos en los lag -2 y -1 respectivamente, finalizando las acciones en ZFF (zona de finalización F), correspondiente al espacio ocupado por el extremo izquierdo -figura 1-. Sousa, Prudente, Sequeira, LópezLópez y Hernández-Mendo (2015) en su estudio de situaciones de juego 2x2 en el campeonato europeo masculino de balonmano 2012, determinaron que el cruce, como elemento técnico-táctico, activa de forma significativa el lanzamiento de 7 metros; mientras que la acción técnico-táctica de bloqueo activa la consecución de gol.

El 2x2 es un elemento técnico-táctico que forma parte de los medios tácticos básicos (Foretić, Rogulj y Trninic, 2010; García, Aníz, Arellano, Domínguez, y García, 2004; Montoya, Moras y Anguera, 2013; Rogulj, Srhoj, y Srhoj, 2004; Román, 2005). Por ello resulta relevante destacar la ausencia de asociación significativa entre las conductas criterio "exitosas" -GOL (consecución del gol) y 7M (lanzamiento de 7 metros a favor)- y la categoría 2x2 (X22) -dimensión creación- en el desarrollo de la secuencia ofensiva. Estos resultados difieren con los obtenidos por Prudente et al. (2017), en balonmano masculino, quienes concluyen que las situaciones de $2 \times 2$ poseen una gran importancia táctica a lo largo del desarrollo de un encuentro. También con Passos, Milho, Fonseca, Borges, Araújo y Davids (2011), quienes en su estudio acerca de los deportes de equipo, afirman que las tendencias de agrupación, en el juego colectivo ofensivo, se reducen en subunidades de dos individuos, siendo sensibles a diferentes delimitaciones de tareas, como son, en este caso, las acciones de juego que se producen en situaciones de asimetría numérica favorable.

Los resultados obtenidos en cuanto a las acciones ofensivas que finalizan con un lanzamiento que no acaba en gol (ML) muestran una asociación estadísticamente significativa con el inicio de secuencia en forma saque de 
centro (SC), y mediante sistemas tácticos estructurados (STE). Estos resultados difieren con los ya señalados de Lozano y Camerino (2012). En el desarrollo y finalización de la secuencia ofensiva se ha detectado una relación de asociación estadísticamente significativa entre la conducta criterio lanzamiento que no acaba en gol (ML) y las conductas condicionadas cruce (CR) y la zona de finalización A (véase figura 1) en el lag -2 y la coocurrencia respectivamente. Estos resultados coinciden con los obtenidos por Sousa et al. (2015), quienes concluyen en su estudio de las situaciones de juego $2 \times 2$, en el campeonato europeo masculino de balonmano 2012, que el "cruce y continuidad" activa el lanzamiento al poste o al travesaño.

En cuanto a las secuencias ofensivas que finalizan con una pérdida de balón por parte del equipo observado (PBL), destacar la relación de activación estadísticamente significativa en el lag 0 o coocurrencia con la situación táctica de prefinalización 4vs3 (VS43); también se produce una relación estadísticamente significativa de inhibición en la coocurrencia de la situación 1vs0 (VS01). Se pone de manifiesto que las secuencias en las que se realizan situaciones tácticas complejas, en las que se agrupen más de dos o tres jugadores, tienen mayor dificultad de finalizar con éxito (Sousa et al. 2015).

\section{CONCLUSIÓN}

A la luz de los resultados obtenidos en el presente trabajo, se puede concluir que el éxito de las secuencias ofensivas en situaciones de superioridad numérica favorable, durante el campeonato mundial de balonmano femenino de 2016, responde a un patrón de juego ofensivo que utiliza un sistema táctico no estructurado, sin ser relevante su forma de inicio (robo de balón en situaciones de 1x1 ○ 1x2, interceptación del balón, saque de puerta, saque de centro, infracción reglamentaria por parte del equipo rival o pérdida de balón del equipo rival), que emplea como principal medio ofensivo el contraataque de primera oleada, para finalizar las acciones con situaciones de $1 \mathrm{vs} 0$.

El presente estudio pretende ayudar a los entrenadores de balonmano femenino a profundizar en el conocimiento de las secuencias ofensivas con superioridad numérica derivadas de una sanción disciplinaria de exclusión (dos minutos de juego en inferioridad numérica) en el balonmano de élite femenino. Sirviendo como punto de apoyo de cara al diseño de tareas, orientadas a la simulación de situaciones de contraataque con situaciones de 2x2 como medio para alcanzar un lanzamiento; o bien, que permita aplicar medios tácticos de menor complejidad de ejecución para la consecución del gol.

\section{REFERENCIAS BIBLIOGRÁFICAS}

Anguera, M. T., Blanco-Villaseñor, A. y Losada, J. L. (2001). Diseños observacionales, cuestión clave en el proceso de la metodología observacional. Metodología de las Ciencias del Comportamiento, 3(2), 135-160.

Anguera, M.T. (1979) Observational Typology. Quality \& Quantity. EuropeanAmerican Journal of Methodology, 13(6), 449-484. 
Anguera, M. T. y Hernández-Mendo, A. (2013). La metodología observacional en el ámbito del deporte. E-balonmano.com: Revista de Ciencias del Deporte, 9(3), 135-160.

Anguera, M. T., y Hernández-Mendo, A. (2015). Técnicas de análisis en estudios observacionales en ciencias del deporte. Cuadernos de Psicología del Deporte, 15(1), 13-30.

Anguera, M. T., Magnusson, M. S. y Jonsson, G. K. (2007). Instrumentos no estándar. Avances en Medición, 5(1), 63-82.

Antúnez, A., García, J, Sáez, F. J., Valle, A. y García, A, (2013). Diferencias en los indicadores de rendimiento entre equipos ganadores y perdedores en etapas de formación en balonmano en función del género y la diferencia final de goles. E-balonmano.com: Revista de Ciencias del Deporte, 9(1), 5-16.

Asker, M., Waldén, M., Källberg, H., Holm, L. W. y Skillgate E. (2017) A prospective cohort study identifying risk factors for shoulder injuries in adolescent elite handball players: the Karolinska Handball Study (KHAST) study protocol. BMC Musculoskeletal Disorders, 18:485. DOI 10.1186/s12891-017-1852-2.

Bakeman, R. y Quera, V. (1995). Analyzing interaction: Sequential analysis with SDIS and GSEQ. Cambridge: Cambridge University Press.

Bakeman, R. y Quera, V. (2011). Sequential Analysis and Observational Methods for the Behavioral Sciences. Cambridge: Cambridge University Press.

Cardinale, M., Whiteley, R., Hosny, A. A. y Popovic, N. (2017). Activity Profiles and Positional Differences of Handball Players During the World Championships in Qatar 2015. International Journal of Sports Physiology and Performance, 12 (7), 908-915. DOI: 10.1123/ijspp.2016-0314.

Cohen, J. (1960). A coefficient of agreement for nominal scales. Educational and Psychological Measurement, 20, 37-46.

Debanne, T., Laffaye, G. y Trouilloud, D. (2018). Motivational orientations and performance in penalty throws during elite male team handball games. Scandinavian Journal of Medicine \& Science in Sports, 28(3), 1288-1294. DOI: $10.1111 / \mathrm{sms} .12995$.

Dello lacono, A., Martone, D., Zagatto, A. M., Meckel, Y., Sindiani, M., Milic, M. y Padulo, J. (2018). Effect of contact and no-contact small-sided games on elite handball players. Journal of Sports Sciences. 36 (1), 14-22. DOI:10.1080/02640414.2016.1276296.

Espina, J. J., Pérez, J. A. y Cejuela, R. (2012). Evolución histórica y táctica de los sistemas de juego defensivos en balonmano en situaciones de desigualdad numérica. E-balonmano.com Revista de Ciencias del Deporte, 8(2), 93-104.

Ferrari, W. R., dos Santos, J. V. y Vaz, V. P. S. (2014). Offensive Process Analysis in Handball: Identification of Game Actions that Differentiate Winning from Losing Teams. American Journal of Sports Science, 2(4), 92-96. doi: 10.11648/j.ajss.20140204.14.

Fieseler, G., Hermassi, S., Hoffmeyer, B., Schulze, S., Irlenbusch, L., Bartels, T., Delank, K. S., Laudner, K. G. y Schwesig, R. (2017). Differences in anthropometric characteristics in relation to throwing velocity and competitive level in professional male team handball: a tool for talent 
profiling. Journal of Sports Medicine and Physical Fitness, 57(7-8), 985992. DOI:10.23736/S0022-4707.17.06938-9.

Fleiss, J. L., Levin, B. y Paik, M. C. (2003). Statistical methods for rates and proportions, 3rd ed. Hoboken: John Wiley y Sons.

Foretić, N., Rogulj, N. y Trninić, N. (2010). The influence of situation efficiency on the result of a handball match. Sport Science. Faculty of Kinesiology, 2, 45-51.

Forthomme, B., Croisier, J. L. Delvaux, F., Kaux, J. F., Crielaard, J. M. y GleizesCervera, S. (2018). Preseason Strength Assessment of the Rotator Muscles and Shoulder Injury in Handball Players. Journal of Athletic Training, 53(2), 174-180. DOI: 10.4085/1062-6050-216-16.

Gabin, B., Camerino, O., Anguera, M. T. y Castañer, M. (2012). Lince: Multiplatform sport analysis software. Procedia. Social and Behavioral Sciences, 46, 4692-4694.

García, J. A., Aníz, I., Arellano, J. I., Domínguez, J.O. y García, T. (2004). Influencia de las variables tiempo y distancia en la eficacia del juego con transformaciones en cuatro equipos de balonmano de alto nivel. Posibilidades para la aplicación en el entrenamiento. Motricidad. European Journal of Human Movement, 12, 79-94.

González, I. (2015). El uso táctico del golpe franco en el balonmano. Ebalonmano.com: Revista de Ciencias del Deporte. 11(1), 39-54.

Grabara, M. (2018). The posture of adolescent male handball players: A two-year study. Journal of Back and Musculoskeletal Rehabilitation, 31(1), 183-189. DOI: 10.3233/BMR-170792.

Gutiérrez, D., Fisette, J., García-López, L. M. y Contreras, O. (2014). Assessment of secondary school students' game performance related to tactical contexts. Journal of Human Kinetics, 42(1), 223-234. DOI: 10.2478/hukin-2014-0076.

Gutiérrez, O., Fernández, J. J. y Borrás, F. (2010). Uso de la eficacia de las situaciones de juego en desigualdad numérica en balonmano como valor predictivo del resultado final del partido. E-balonmano.com Revista de Ciencias del Deporte, 6(2), 67-77.

Hermassi, S., Chelly, M. S., Fieseler, G., Bartels, T., Schulze, S., Delank, K. S., Shephard, R. J. y Schwesig, R. (2017). Effects of In-Season Explosive Strength Training on Maximal Leg Strength, Jumping, Sprinting, and Intermittent Aerobic Performance in Male Handball Athletes. Sportverletzung-Sportschaden, 31(3), 167-173. DOI: 10.1055/s-0043103469.

Hernández, J. M., Rodríguez, A. J., Hernández, J., Álvarez, P. A., Jiménez, F. y Hernández, I. M. (2010). Análisis del juego de ataque en balonmano femenino. Ágora, para la educación física y el deporte, 12(3), 257-272.

Hernández-Mendo, A., Castellano, J., Camerino, O., Jonsson, G., BlancoVillaseñor, Á., Lopes, A. y Anguera, M. T. (2014). Programas informáticos de registro, control de calidad del dato, y análisis de datos. Revista de Psicología del Deporte, 23(1), 111-121.

Hristovski, R., Aceski, A., Balague, N., Seifert, L., Tufekcievski, A. y Cecilia, A. (2017). Structure and dynamics of European sports science textual contents: Analysis of ECSS abstracts (1996-2014). European Journal Sport Science, 17, 19-29. DOI:10.1080/17461391.2016.1207709. 
Ingebrigtsen, J., Jeffreys, I. y Rodahl, S. J. (2013). Physical characteristics and abilities of junior elite male and female handball players. Journal of Strength and Conditioning Research, 27(2), 302-309. Doi: 10.1519/JSC.0b013e318254899f.

Lopiano, D. A. (2000). Modern history of women in sports: Twenty-five years of Title IX. Clinics in Sports Medicine, 19(2), 163-173.

Lozano, D. (2014). Análisis del comportamiento táctico ofensivo en alto rendimiento en balonmano (Tesis doctoral). Universitat de Lleida. Lleida. España.

Lozano, D. y Camerino, O. (2012). Eficacia de los sistemas ofensivos en balonmano. Apunts. Educación Física y Deportes, 108(2), 70-81 DOI: 10.5672/apunts.2014-0983.es.(2012/2).108.08.

Lozano, D., Camerino, O. e Hilenio, R. (2016). Interacción dinámica ofensiva en balonmano de alto rendimiento. Apunts. Educación Física y Deportes, 125(3), 90-110. DOI: http://dx.doi.org/10.5672/apunts.20140983.es.(2016/3).125.08.

Lubiatowski, P., Kaczmarek, P., Cisowski, P., Breborowicz, E., Grygorowicz, M., Dzianach, M., Krupecki, T., Laver, L. y Romanowski, L. (2018). Rotational glenohumeral adaptations are associated with shoulder pathology in professional male handball players. Knee Surgery Sports Traumatology Arthroscopy, 26 (1), 67-75. DOI: 10.1007/s00167-017-4426-9.

Mayer, J. y Thiel, A. (2018). Presenteeism in the elite sports workplace: The willingness to compete hurt among German elite handball and track and field athletes. International Review for the Sociology of Sport. 53 (1), 4968. DOI: $10.1177 / 1012690216640525$.

Menezes, R. P., dos Reis, H. H. B. y Tourinho, H. (2015). Teaching-learningtraining of individual technical-tactical elements of handball for Under-14, Under-16 and Under-18 teams. Movimento, 21(1), 261-273.

Mhenni, T., Michalsik, L. B., Mejri, M. A., Yousfi, N., Chaouachi, A., Souissi, N. y Chamari, K. (2017). Morning-evening difference of team-handballrelated short-term maximal physical performances in female team handball players. Journal of Sports Science, 35(9), 912-920. Doi: 10.1080/02640414.2016.1201212.

Michalsik, L. B., Aagaard, P. y Madsen, K. (2013). Locomotion Characteristics and Match-Induced Impairments in Physical Performance in Male Elite Team Handball Players. International Journal of Sports Medicine, 34(7), 590-599. DOI: 10.1055/s-0032-1329989.

Michalsik, L. B., Madsen, K. y Aagaard P. (2015). Physiological capacity and physical testing in male elite team handball. Journal of Sports Medicine and Physical Fitness, 55(5), 415-29.

Montoya, M., Moras, G. y Anguera, M. T. (2013). Análisis de las finalizaciones de los jugadores extremo en balonmano. Apunts. Educación Física y Deportes, 113, 52-59.

Morillo, J. P. y Hernández-Mendo, A. (2015). Análisis de la calidad del dato de un instrumento para la observación del ataque en balonmano playa Revista Iberoamericana de Psicología del Ejercicio y el Deporte, 10(1), 15-22.

Morillo-Baro, J. P., Reigal, R. E. y Hernandez-Mendo, A. (2016). The relationsship between the sports psychological profile and competitive 
anxiety in beach handball players. Revista de Psicologia del Deporte, 25(1), 121-128.

Navarro, A., Morillo, J. P., Reigal, R. E. y Hernández-Mendo, A. (2018). Polar coordinate analysis in the study of positional attacks in beach handball. International Journal of Performance Analysis In Sport, 18(1), 151-167. DOI: $10.1080 / 24748668.2018 .1460052$.

Ntai, A., Zahou, F., Paradisis, G., Smirniotou, A. y Tsolakis, C. (2017). Anthropometric parameters and leg power performance in fencing. Age, sex and discipline related differences. Science \& Sports, 32(3), 135-143.

Ohnjec, K., Vuleta, D., Milanović, D. y Gruić, I. (2008). Performance indicators of teams at the 2003 world handball championship for women in croatia. Kinesiology, 40(1), 69-79.

Passos, P., Milho, J., Fonseca, S., Borges, J., Araújo, D. y Davids, K. (2011). Interpersonal distance regulates functional grouping tendencies of agents in team sports. Journal of Motor Behavior, 43(2), 155-163. doi: 10.1080/00222895.2011.552078.

Prieto, J., Gómez, M. A. y Sampaio, J. (2015). From a Static to a Dynamic Perspective in Handball Match Analysis: a Systematic Review. The Open Sports Sciences Journal, 8, 25-34.

Prudente, J., Sousa, D., Sequeira, P., López-López, J. A. y Hernández-Mendo, A. (2017). Analyzing the influence of playing time and partial score on the tactical behavior in the duel 2 vs 2 in the offensive process in handball, using the polar coordinates technique. Anales de psicología, 33(3), 515529. DOI: http://dx.doi.org/10.6018/analesps.33.3.271071.

Pueo, B., Jiménez-Olmedo, J. M., Penichet-Tomas, A., Ortega, M. y Espina, J. J. (2017). Analysis of Time-Motion and Heart Rate in Elite Male and Female Beach Handball. Journal of Sports Science and Medicine, 16, 450-458.

Ribeiro, P., Oliveira, M. C., Freitas, O., Leitão, C., Anguera, M. T. y Campaniço, J. (2009). The methodology observational in Handball: Review of offensive actions of selection champion in Europe in 2008. Motricidade, 5(3), 95.

Rogulj, N., Srhoj, V. y Srhoj, L. (2004). The contribution of collective attack tactics in differentiating handball score efficiency. Collegiums Antropologicum, 28(2), 739-746.

Román, J. D. (2005). Conceptos de ataque frente a variantes defensivas 6:0 y 5:1. E-balonmano.com: Revista de Ciencias del Deporte, 1, 3-16.

Román, J. D. (2008). Táctica colectiva grupal en ataque: los modelos en el balonmano español. E-balonmano.com: Revista de Ciencias del Deporte, 4 (2), 29-51.

Sabido, R., Hernández-Davo, J. L., Botella, J., Navarro, A. y Tous-Fajardo, J. (2017). Effects of adding a weekly eccentric-overload training session on strength and athletic performance in team-handball players. European Journal of Sport Science, 17(5), 530-538. DOI: 10.1080/17461391.2017.1282046.

Sousa D. J., Prudente J. N., Sequeira P., López-López J. A. y Hernández-Mendo A. (2015). Análisis de las situaciones de juego $2 \times 2$ en el campeonato masculino de balonmano 2012: Aplicación de la técnica de coordenadas polares. Cuadernos de psicología del deporte, 15(1), 181-194. 
Srhoj, V., Rogulj, N., Padovan, M. y Katić, R. (2001). Influence of the attack end conduction on match result in handball. Collegiums Antropologicum, 25(2), 611-7.

Steffen, K., Nilstad, A., Krosshaug, T., Pasanen, K., Killingmo, A. y Bahr, R. (2017). No association between static and dynamic postural control and ACL injury risk among female elite handball and football players: a prospective study of 838 players. British Journal of Sports Medicine, 51(4), 253-259. Doi: 10.1136/bjsports-2016-097068.

Wagner, H., Finkenzeller, T., Würth, S. y von Duvillard, S. P. (2014). Individual and Team Performance in Team-Handball: A Review. Journal of Sports Science and Medicine, 13, 808-816.

Wagner, H., Gierlinger, M., Adzamija, N., Ajayi, S., Bacharach, D.W. y von Duvillard, S. P. (2017). Specific Physical Training In Elite Male Team Handball. The Journal of Strength and Conditioning Research, 31(11), 3083-3093. DOI: 10.1519/JSC.0000000000002094.

Número de citas totales/Total references: 60 (100\%)

Número de citas propias de la revista / Journal's own references: $0(0 \%)$

Rev.int.med.cienc.act.fís.deporte - vol. 20 - número 78 - ISSN: 1577-0354 